\title{
INTENSITY-BASED SIGNAL SEPARATION ALGORITHM FOR ACCURATE QUANTIFICATION OF CLUSTERED CENTROSOMES IN TISSUE SECTIONS
}

\author{
Markus C. Fleisch, Christopher A. Maxwell, Claudia K. Kuper, Erika T. Brown, \\ Mary Helen Barcellos-Hoff, Sylvain V. Costes ${ }^{\wedge}$
}

Life Sciences Division, Lawrence Berkeley National Laboratory, Berkeley, CA

\author{
${ }^{\wedge}$ Corresponding Author: Sylvain V. Costes, $\mathrm{PhD}$ \\ Life Sciences Division, \\ 1 Cyclotron Road, MS 977-225A \\ Lawrence Berkeley National Laboratory \\ Berkeley CA 94720 \\ Phone: (510) 486-6988 \\ Fax: (510) 486-5586 \\ Email: SVCostes@lbl.gov
}

Funding Support: This work was funded by the Low Dose Radiation Research Program, Office of Biological Effects Research, Health Effects Division, United States Department of Energy (contract no.03-76SF00098). CAM was supported by a postdoctoral multidisciplinary award (BC050612) from the Department of Defense Breast Cancer Research Program (BCRP).

Running Title: Centrosome quantification in tissue sections

Key Words: Centrosome, quantification, microscopy, segmentation, immunofluorescence, p53

Word Count [excluding references]: 3650

Pages: 21

Tables: 0

Figures: 6 


\begin{abstract}
Centrosomes are small organelles that organize the mitotic spindle during cell division and are also involved in cell shape and polarity. Within epithelial tumors, such as breast cancer, and some hematological tumors, centrosome abnormalities (CA) are common, occur early in disease etiology, and correlate with chromosomal instability and disease stage. In-situ quantification of CA by optical microscopy is hampered by overlap and clustering of these organelles, which appear as focal structures. CA has been frequently associated with Tp53 status in premalignant lesions and tumors. Here we describe an approach to accurately quantify centrosome frequencies in tissue sections and tumors, independently of background or noise levels. Applying simple optical rules in non-deconvolved conventional 3D images of stained tissue sections we show that we can evaluate more accurately and rapidly centrosome frequencies than with traditional investigator based visual analysis or threshold-based techniques. The resulting population-based frequency of centrosomes per nucleus can then be used to approximate the proportion of cells with $\mathrm{CA}$ in that same population. This is done by taking into account baseline centrosome amplification and proliferation rates measured in the tissue. Using this technique we show that $20-30 \%$ of cells have amplified centrosomes in $T p 53$ null mammary tumors.
\end{abstract}




\section{INTRODUCTION}

Centrosomes are small organelles that nucleate microtubules, coordinate mitotic spindle assembly and, thus, are critical for correct chromosome segregation during mitosis. They are involved in many other cellular features involving microtubules like shape and cell polarity. Concurrent with DNA synthesis, centrosomes replicate once during the cell cycle in preparation for the generation of a bipolar spindle. Thus, depending on the cell cycle phase, a normal somatic cell has either 1 (G0-1) or 2 (G2-S) centrosomes. Within human tumors, the ubiquitous presence of centrosomal amplification, consisting of supernumerary (greater than two) centrosomes and/or centrosomes of aberrant size and structure, has been implicated in the generation of aneuploidy and carcinogenesis with recent studies showing that in-situ centrosome amplification is a frequent event in many cancers and precancerous lesions (Pihan and others, 1998; Pihan and others, 2003). Within tumors of epithelial and hematological origin, such as breast cancer and multiple myeloma, centrosomal amplification is common, occurs early in disease etiology, correlates with chromosomal instability and disease stage and is associated with poor prognostic genetic and clinical subtypes (Chng and others, 2005; Lingle and others, 2002; Maxwell and others, 2005; Pihan and others, 1998; Salisbury and others, 2004). For these reasons, the analysis of in-situ centrosomal amplification within premalignant tissues may provide significant insight into tumor prognosis, progression and outcome.

Various ways have been used to quantify centrosomes in biological specimen mostly using confocal microscopy or deconvolution (Dodson and others, 2004; Goepfert and others, 2000; Li and others, 2004; Lingle and others, 2002). As the position of two 
centrosomes can vary within the cytoplasm, three dimensional imaging and analysis is mandatory but cumbersome and time consuming. When analyzing cells grown in monolayers some investigators have used a cell based manual count approach presenting the fraction of cells with an abnormal centrosome number $(>2)$ (Figure 1A). This form of analysis is precise but time consuming and potentially biased by the investigator. Merging microscopy with automated image analysis allows a non-biased quantification of biological objects, such as cells, nuclei or cellular organelles. Most automated approaches use segmentation. A variety of segmentation methods have been developed over the years (for review see (Fernandez-Gonzalez and others, 2004)), typically based on looking at the intensity distribution of an image to separate it into two main classes: the background and the foreground. However, no one segmentation method will work for all images and segmentation results are often imperfect. There are many situations under which most techniques fail and in such situations, manual counting by visual inspection remains the only quantitative alternative. Automated segmentation techniques combining nuclear and centrosome segmentation have been developed in our laboratory allowing an assignment of individual centrosomes to individually segmented nuclei in cultured cells (Figure 1B)(Raman and others, 2005). However, this approach is difficult and technically questionable to apply to analysis of centrosomes in situ using cryosections because of the difficulty in accurately segmenting individual nuclear areas. Immunostaining of thin tissue cryosections (approximately $5 \mu \mathrm{m}$ ) leads to a considerable amount of overlap and apparent clustering of centrosomes which makes their segmentation also challenging. Thus, the assignment of individual centrosomes to the corresponding nucleus is virtually impossible and does not allow a reliable per cell based analysis (Figure 1C and D). To 
avoid this problem other investigators have reported the average number of centrosomes per nucleus in a field per view (Li and others, 2004; Maxwell and others, 2005).

In this paper we present a novel and simple maximum intensity projection (MIP) based-method to quantify centrosomes and other small cellular organelles that failed to be segmented accurately by standard segmentation approaches. After validation by computational simulation, the approach highlights the presence of aberrant centrosomes within Tp53 null transplanted murine tumors, which were previously reported to have low or no CA (Goepfert and others, 2000).

\section{Material and Methods}

\section{Immunofluorescence protocol}

Inguinal $\left(4^{\text {th }}\right)$ mouse mammary glands from adult Balb/c mice and mammary tumors derived from transplanted $T p 53$ null mouse mammary epithelium were dissected free of the skin and embedded in OCT compound (Miles Inc., Elkhart, IN). Frozen embedded mammary glands and tumors were sectioned at $4-5 \mu \mathrm{m}$ onto gelatin-coated coverslips and fixed using $100 \%$ ice cold $\mathrm{MeOH}$ for 10 minutes. Nonspecific sites were blocked using the supernatant from a $0.5 \%$ casein/PBS solution ( $\mathrm{pH} 7.4)$ for 60 minutes. Sections were incubated with a polyclonal rabbit anti-pericentrin antibody (Covance, PRB-432C) and/or a rat anti-Ki67 (DAKO, M-7249) antibody diluted in $0.5 \%$ casein/PBS solution overnight at $4{ }^{\circ} \mathrm{C}$. The antibody and similar staining protocols have been used in a number of studies on centrosomes in human and murine tissues (Lingle and others, 2002). In our studies, as well as in most studies published by other groups, staining results gained with 
pericentrin antibody were confirmed by performing a dual staining with other known centrosomal markers like gamma-tubulin or centrin, which show perfect co-localization. The next day, sections were washed and incubated in a goat anti-rabbit Texas Red and/or a goat anti-rat FITC secondary antibody (Molecular Probes) for 1 hour at room temperature. Nuclei were counterstained with 4',6-diamino-2-phenylindole (DAPI) and mounted with Vectashield (Vector Laboratories). All stainings were performed in triplicates.

\section{Image acquisition}

Tissues were viewed and imaged using a Zeiss Axiovert epifluorescence microscope (Carl Zeiss, Jena, Germany) equipped with a multiband pass filter and a differential wavelength filter wheel. Images were acquired using a Zeiss plan-apochromat $63 \mathrm{X}$ oil, with a NA of 1.25 and a scientific-grade 12-bit charged coupled device camera (ORCA AG Hamamatsu, $6.45 \times 6.45 \mu \mathrm{m}^{2}$ pixels). The image pixel size was measured to be $0.1 \mu \mathrm{m}$ but based on the NA of the objective, the actual resolution of the image in the FITC channel is $\sim 0.5 \times 0.488 / \mathrm{NA}=0.19 \mu \mathrm{m}$. All images were captured with the same exposure time so that intensities were within the 12-bit linear range. $Z$ stacks were taken with a step of $0.25 \mu \mathrm{m}$ and for investigator based visual counting images were deconvolved using blind deconvolution with Autoquant (AutoQuant Imaging Inc., Troy, NY). On the other hand, our method was applied directly on non-deconvolved images.

\section{Image processing and analysis}

Image algorithm was developed under the DipImage imaging platform (Delft University of Technology, the Netherlands) for MATLAB (The Mathworks, Natick, 
MA). As we were particularly interested in the centrosome frequency of luminal epithelial and not the surrounding stroma cells we defined a region of interest (ROI) by manually circling the luminal epithelial cell layer and cropping the projected images according to this ROI. Nuclei were counted in the cropped best focus projection of the DAPI image using the "manually count objects" (MCO) function in MetaMorph ${ }^{\circledR}$. For comparative analysis the cropped red channel (Texas Red) was subjected to a summatory intensity projection (SIP) or maximum intensity projection (MIP) on the raw ${ }^{1}$ image. Projection is a way to visualize 3D images along a given direction. Once the direction is chosen, parallel lines to this axis are used to integrate the intensity content of the 3D images into a single 2D plane. Pixel intensities found in each of these parallel lines can be integrated in different ways: either by summing the intensity of all the pixels along each parallel lines (i.e. summatory intensity projection (SIP)) or by picking only the brightest pixel along the line (i.e. maximum intensity projection (MIP)). In biomedical imaging, MIP is typically used to visualize rotating $3 \mathrm{D}$ objects, by constantly updating the projection of the $3 \mathrm{D}$ stack along the direction linking the center of the object to the surface of the screen, mimicking the way an object would be observed in the real world. In our case, we defined the $\mathrm{Z}$ axis of the microscope as our projection axis, greatly simplifying the computation.

Bead-looking like images were simulated by convolving one pixel wide positive signals with a Gaussian filter of radius similar to the microscope we used. Background was simulated by adding a Gaussian noise of mean representative of real images and standard deviation equals to $0.6 \%$ of the background mean.

\footnotetext{
${ }^{1} \mathrm{~A}$ raw image is an image that has not been modified or corrected by any imaging processes after acquisition.
} 
The threshold for each image was either set manually ("manually threshold object" function MetaMorph ${ }^{\circledR}$ ) by the investigator when analyzing real tissue or obtained automatically by isodata thresholding in the case of simulated images. With this threshold, the operator defined a region of interest representative of single centrosomes in various ways (i.e. visually or automatically using size range as criterion). For investigator based centrosome counts the cropped Texas Red image was deconvolved and counts were performed on consecutive z-stacks again using the MCO function. Results were compared in a $\mathrm{x}-\mathrm{y}$ dot plot and level of correlation $\left(\mathrm{R}^{2}\right)$ was computed in EXCEL (Microsoft ${ }^{\circledR}$ Excel 2002).

Proliferation rate was determined for each individual animal by visually quantifying the fraction of Ki67 positive cells using the text annotation function of Corel PhotoPaint ${ }^{\circledR}$ Version 7.

\section{Calculating fraction of abnormal cells}

The average number of centrosomes per cell we measure in a population of cells is as follows:

$$
I=n+2 p+a A=n+2 p+(1-n-p) A
$$

Where $n$ is the fraction of normal non-proliferating cells, $p$ is the fraction of normal proliferating cells in $\mathrm{S} / \mathrm{G} 2$ (Ki67), $a$ is the fraction of abnormal cells and $A$ is the average number of centrosomes per abnormal cell (Figure $5 \mathrm{~A}$ and $\mathrm{B}$ ). Visual estimation of $\mathrm{A}$ in different cell populations (tumor or normal tissue) and in vitro results of populations with CA suggest little fluctuation (data not shown), with the average number of centrosomes in an abnormal cell primarily being between 3 and 4 . Assuming $A$ is a constant, Eq. 1 can 
then be used to approximate the fraction of normal and abnormal cells by measuring the average number of centrosome per cell $I$ and the proliferating fraction $p$ as follow:

$$
n=\frac{I-A-(2-A) p}{1-A} \text { and } a=1-n-p=\frac{1-I+p}{1-A}
$$

\section{RESULTS}

\section{Theoretical approach}

Accurate quantification of centrosomes and other focal biologic signals is a difficult problem in image analysis. The commonly used approach to visually count these cells in tissue sections or images is labor-intensive, potentially biased, technically questionable and not suitable for a routine clinical application. In this report we present an intensity based approach we developed to accurately quantify centrosomes on a population basis. The approach is based on a simple principle of optics: any fluorescent signal collected by light microscopy is the result of the original signal convoluted by the point-spread function of the microscope (neglecting noise for now). In other words, the original signal is "blurred" during acquisition leading to a loss of spatial resolution. Deconvolution algorithm reduces this loss by reversing the "blurring" effect of the point-spread function via the usage of sophisticated mathematical operators such as Fourier Transform. However, even deconvolved images still have a limited resolution dictated by the wavelength of the light itself used to sample the specimen (i.e. resolution $\sim 0.5 \times \lambda / \mathrm{NA}$, see material and method) and the noise in the image. Point signals, much smaller than the resolution of the microscope used, can represent clusters of a given protein. The signal intensity of such a cluster is directly proportional to the amount of protein in that cluster. One aspect in the convolution process is the fact that the total intensity is invariant from 
such process. Consequently, even though a cluster of proteins will look larger than it is once acquired by light microscopy its total intensity remains unchanged (i.e. still neglecting noise and background).

To illustrate further this point, we generated a known number of bead-like objects $(n=265$, Figure 2) and counted them using different methods. A Gaussian filter was used to convolve one-pixel wide theoretical events with a constant intensity of 1 (Figure 2A) to simulate the point-spread function measured in real images (Figure 2B). The high density of the initial events leads to high amount of clustering of these objects, hampering classical threshold-based counting approaches such as triangle, isodata, or backgroundsymmetry algorithms (Ridler and Calvard, 1978; Zack and others, 1977). As shown in Figure $2 \mathrm{C}$-E, these threshold approaches are clearly inadequate for object identification, resulting in this case with a 10 -fold lower count from the actual number of events. Visual examination, based on two investigator's counts, is not ideal either since in this case it led to a $40 \%$ loss of the actual number of events. On the other hand, the total intensity of the image remains constant after blurring (Figure 2B), showing that total intensity is an invariant property of an image reflecting the proportionality to the number of objects in the image. By knowing the intensity value of one object in the image, one can then calculate the number of objects in the image by dividing the total intensity of the image by the unit intensity value (i.e. referred to as the normalization step).

However, this normalization step clearly depends on noise and background variation in real images. Correcting for these factors is difficult as noise varies from image to image and background is not always uniform throughout an image. A robust way to remove variation due to background and noise fluctuation is to identify regions within 
each image with specific signal and apply the intensity normalization step to these regions instead of the full field of view. In this refined method, approximate spot segmentation is obtained by thresholding the image. This is done by either the operator visually selecting a threshold level or by automatic thresholds (Figure 3B). In the case of automatic threshold methods, isodata-thresholded images (method previously mentioned) gave the highest counts for threshold-based segmentations leading to an underestimation of about $40 \%$ (Figure 3C). On the other hand, quantifying the total intensity within the segmented sub-regions and normalizing by the average total intensity of a single spot (Figure 3B, blue circles), results in less than $5 \%$ deviation from the true value (Figure 3D). In addition, no noticeable difference is observed in the accuracy of the results for a variation of signal to noise ratio ranging between 2 and 0.5 (Figure 3D).

We then evaluated the validity of our approach by comparing centrosome counts on actual tissue specimen immunostained for centrosomes to visual quantification made by an investigator in our laboratory. Figure 4A shows such a specimen in which centrosomes clusters are typical and consistently underscored by thresholding techniques. Even an investigator based visual count can be difficult in cases of intense clustering of events (Figure 4B). The presented approach detects automatically from each thresholded image reference single objects (i.e. detection based on the expected range size for single centrosomes) and computes their average total intensity (Figure 4A and 4B show some example of detected single objects, circled in blue). This mean intensity is then used to normalize the total intensity of the segmented full field of view. By doing so, the number of centrosome per field of view is highly correlated to visual inspection for normal tissue (Figure 4C-E). The intensity-based analysis on MIP of the raw image correlated better 
with the investigators counts $\left(\mathrm{R}^{2}=0.8, \mathrm{y}=0.94 \mathrm{x}\right)$ than $3 \mathrm{D}$ analysis $\left(\mathrm{R}^{2}=0.74\right)$ or SIP based approaches $\left(\mathrm{R}^{2}=0.34\right)$. In addition, as previously shown in Figure 3 with simulated images, counting centrosomes simply by segmenting the image using a visual threshold leads to counts well correlated but much lower than visual inspection ( 25 to $50 \%$ lower).

As the investigator based threshold might be another potential source of variation in our results we simulated different threshold levels on a blurred image with a defined number of beads (Figure 5A-D). We found that within a wide range of thresholds (in our example range was between 75 and 110 and single spot center had an intensity equal to 130) the relative error remained in an acceptable range below 4\% (Figure 5D). Outside this range, using a very low or very high threshold value led to an underestimation or over-prediction of counts, respectively. This is illustrated in Figure 5E, as the relative error (absolute value) increases rapidly in both such extreme cases.

To further test the robustness of our algorithm in a real case scenario, we evaluated its ability to discriminate between normal and tumor tissue by comparing their centrosome abnormality frequencies. Immunostained cryosections of normal mouse mammary gland and mouse mammary tumors derived from a $T p 53$ null mammary epithelial outgrowth line were analyzed for that purpose. After subtraction of the underlying proliferation rate (Ki67), which was $4.8 \%$ for the normal tissue and $9.6 \%$ for the tumors, we found an average of 1.07 centrosomes for normal mammary gland and 1.77 for the tumors. These values led to an approximated fraction of abnormal cells between 0 and 5\% for the normal tissue and between 20 and $30 \%$ for the tumors, assuming a number of centrosomes per abnormal cell to be between 4 and 3, respectively (Figure 6C and D). 


\section{Discussion}

The reported aberrations of centrosomes in a variety of cancers and also precancerous lesions make $\mathrm{CA}$ an interesting diagnostic or prognostic candidate marker for different cancers. A majority of analyses report CA based upon immunofluorescence quantification of pericentriolar material (PCM), generally either gamma-tubulin and/or pericentrin. An advantage of these analyses is that both numerical and structural CA can be determined based upon PCM signals. Structural CA strongly correlate with abnormal mitoses in breast cancer and are related to chromosomal instability in breast tumors and multiple myeloma (Lingle and others, 2002)(Maxwell and others, 2005). A necessary prerequisite for such analysis is a robust staining. This is readily accomplished using various antibodies directed to centrosome components. The preferable measurement for $\mathrm{CA}$ is the fraction of cells in a given tissue with abnormal centrosomes (i.e. $>3$ ). However, quantitative evaluation of CA requires an imaging approach that can deal with the overlap and clustering of centrosomes in tissue. Thus, these challenging staining patterns frequently hamper precise quantification of CA.

In the presented approach, we determine the average number of centrosomes per nucleus in a field of view by determining the number of centrosomes per image normalized to the number of manually counted nuclei. One can then compute an estimate of cells with abnormal centrosomes by careful mathematical consideration of proliferation rate and average number of centrosomes in abnormal cells as described in Material and Methods. One should note that the result is not a fixed value but a range as the actual number of abnormal cells depends on the distribution of the number of centrosomes per abnormal cell. An abnormal cell, by definition, contains a minimum of 3 
centrosomes and analysis of murine mammary epithelial cells ex vivo suggests an average of 3.7 centrosomes per abnormal cell (data not shown).

Our approach is simple and thus rapid for processing large set of data. On the other hand it is not fully automated and still requires the involvement of the investigator at different steps: 1. to define the general area of interest (which in our example was necessary to selectively analyze luminal epithelial cells); 2 . to threshold the image (which is not contributing to any bias); 3. to manually count the nuclei as nuclear segmentation in frozen sections still is a challenge; 4. to identify single centrosomes in each field of view as an intensity reference. The necessary time to perform all these steps for a 40-cell duct in a given image including loading and saving the image was, depending on the investigator, approximately 40-50 seconds. These current limitations can potentially add some bias in the reported results. However, the validity of our method is well illustrated and one can clearly think of ways to fully automatize it. For example, in the tissue data, single centrosomes were in fact automatically identified and used as a reference by considering objects in each thresholded field of view whose size fitted a narrow range (i.e. this size range was visually established as characteristic of single centrosomes over the full dataset). In the course of the analysis we also found that the nuclear area measured on the thresholded DAPI image correlated well with the operator based counts (data not shown) so that a calculation based on nuclear area might be an alternative to visual nuclear counts. A more elaborate nuclear segmentation tool is another alternative. In addition, our simulations in Figure 3 were also performed using an automatic threshold (isodata method) (Ridler and Calvard, 1978). 
One main limitation with this approach is the fact that measurements for individual centrosomes are not available, leading to only population-based measurements. By its nature, this approach is not designed to provide exact numbers of cells with one, two, or three centrosomes. However, as we demonstrate, it provides an accurate approximation of the overall number of centrosomes which allows comparing CA in different tissues. On the other hand, this method could be used to evaluate the potential number of centrosomes in each individual clusters generated by a threshold-based algorithm and thus as a criteria to refine segmentation.

We analyzed three murine breast cancers specimen derived from $T p 53$ null cells. The loss of the tumor suppressor gene $T p 53$, which is frequently mutated in human and murine tumors (Hollstein and others, 1991; Mowat and others, 1985), has been associated with CA and genomic instability (Chiba and others, 2000; Fukasawa and others, 1996; Fukasawa and others, 1997). One study using confocal microscopy on thick tissue sections of mammary tumors from p53 knockout (Tp53 null) mice reported CA only in a small subset of these specimen (Goepfert and others, 2000). Despite the lack of CA in all tumors, all of them exhibited gross genomic instability. The finding that Tp53 null late stage tumors and Tp53 null tumor cell lines exhibit altered but stable karyotypes and normal centrosome behavior had previously been explained by a model of genomic convergence. It is hypothesized that at some point the altered chromosome composition might undergo a selection pressure preferentially selecting for mutations that lead to a stabilization e.g. of the altered centrosome number (Chiba and others, 2000). Using this novel tool, we actually found significant centrosome amplification in $30-40 \%$ of cells in these tumor tissues, indicating the critical role image quantification can play in reporting 
such measurements. This analysis on normal tissue detected $0-5 \%$ of abnormal cells, which is remarkably similar to that obtained by visual inspection of normal human breast tissues (Lingle and others, 2002). The complexity of tissue makes it often difficult for algorithms to quantify imaging features such as centrosome counts and thus pathologists are often doing this type of quantification. However, the accuracy of our estimation of CA in normal tissue is a strong validation of our method and makes the high number of CA we report in tumor tissue more credible.

\section{Summary}

The presented approach allows a precise, partly supervised quantitative analysis of centrosomes in non-deconvolved conventional 3D images of stained tissue sections without the need for background correction and noise consideration. In combination with some calculations, including consideration of proliferation rates, it generates a probability range of cells with $\mathrm{CA}$. A facilitation of centrosome in-situ analysis might help to evaluate $\mathrm{CA}$ as a potential diagnostic and prognostic marker in human and murine malignancies. Although not tested in this study, this approach might well be applicable for other focal staining signals. 


\section{Figure legends}

Figure 1: Centrosome analysis in cell culture vs. tissue sections: A: Immunofluorescence staining for centrosomes (pericentrin, red) and cell cycle marker CENPF (green) on MCF10A mammary epithelial cells. Centrosomes appear as one or 2 separable foci in a variable proximity to an individual nuclei. Examples for abnormal centrosomes $(\uparrow)$ and multipolar spindle $(\uparrow \uparrow)$ are indicated by arrows. B: Nuclear segmentation (DAPI) and segmentation of the centrosome signal (Texas Red) allows an automated assignment of individual centrosomes to specific nuclei (BioQuant software)(Raman and others, 2006). C and D: $4 \mu \mathrm{m}$ frozen sections of a normal mouse mammary duct (C) and a mammary tumor generated from a Tp53 null mammary outgrowth (D). The nature of frozen sections with partly overlapping nuclei and luminal orientation and clustering of centrosomes (see inserts) hampers correct segmentation even when confocal microscopy or deconvolution is used.

Figure 2: Computational simulation of focal staining events and comparison of different projection based approaches: 265 pixels with a theoretical intensity of 1 have been randomly generated and convolved using a Gaussian filter such that they present features of a biological image leading to some bead-looking alike objects which partly cluster (A and B). Convolution of the signal though did not change the total intensity of the image which still equals to 265. C, D and E show the results of different threshold approaches and their corresponding counts if we were to use these masks to identify objects.

Figure 3: Method description. Our method is compared to the isodata-threshold approach for varying object densities and signal to noise ratio $(\mathrm{S} / \mathrm{N})$. (A) Example of one simulated field of view. 105 objects were generated with the same total intensity. (B) Mask of image A obtained by Isodata thresholding. This mask leads to only 75 distinct objects of various sizes, due to the high object density. Two distinct single objects in the mask image (circled in blue) are used as a reference to evaluate the total intensity of a single object in image A. The total intensity of image A in the mask area is then divided by the reference intensity, leading to a value of 103. 10 simulated images with different object densities are analyzed in the same manner for four different $\mathrm{S} / \mathrm{N}$ between 2 and 
0.5. Object counts based on our method and on the isodata-threshold approach are plotted against the real number of object per field of view (panel C and D, respectively). Each color represents a distinct signal to noise ratio.

Figure 4: Correlation of investigator based centrosome counts and results based on different types of analysis. Displayed are magnified segments of in situ mouse mammary gland staining containing centrosome clusters. (A) shows the results of threshold (th) or intensity (i) based analysis in the presented examples. Overlapping signals in the convolved image hamper visual quantification but can be separated by intensity if related to a reference signal (B). Our method was applied to three different representations of non-deconvolved three-dimensional conventional images: (C) the original 3D stack, or the projected stack, using summatory intensity projection MIP (D) or maximum intensity projection SIP (E). In all three cases, number of centrosome measured by threshold segmentation or intensity based counting was compared to investigator visual counts.

Figure 5: Sensitivity of threshold values for intensity-based object counting. Panel A shows the MIP of a simulated 3D image: 200×200x20 pixels, 66 spots (mean intensity 73), Gaussian background (mean 55, standard deviation $0.6 \%$ of mean), signal to noise ratio 1.3. Panel $\mathrm{B}, \mathrm{C}$ and $\mathrm{D}$ shows the resulting masks from the range of thresholds tested on the image in panel A, going from conservative to less conservative (72, 90 and 108 respectively). Panel E shows the relative error in the number of spots measured using different threshold values. Any threshold between 72 and 108 lead to an error on measurement less than $4 \%$ (i.e. 63 to 69 spots measured in that range of thresholds).

Figure 6: A: The centrosome index determined by our method comprises the average of one centrosome per cell for every cell in G0-1 phase. As cells in S and G2 have two centrosomes and this cell cycle phases can be determined by immunoreactivity for Ki67 the index in part reflects the proliferation rate of this population (B). The number of centrosomes above baseline and proliferation rate presents the theoretical excess number of centrosomes. C: Comparison of normal mouse mammary gland tissue sections and Tp53 null mouse mammary gland tumor shows a significant excess of centrosomes. Calculating the theoretical number of abnormal cells for an average of 3 or 4 centrosomes shows that $\mathrm{Tp} 53$ null tumors presumably contain between $29 \%$ and $40 \%$ 
cells with abnormal centrosomes compared to $2.6 \%-3.4 \%$ of cells in the normal population. 


\section{References}

Chiba S, Okuda M, Mussman JG, Fukasawa K. 2000. Genomic Convergence and Suppression of Centrosome Hyperamplification in Primary p53-/- Cells in Prolonged Culture. Experimental Cell Research 258(2):310-321.

Chng WJ, Ahmann GJ, Henderson K, Santana-Davila R, Greipp PR, Gertz MA, Lacy MQ, Dispenzieri A, Kumar S, Rajkumar SV, Lust JA, Kyle RA, Zeldenrust SR, Hayman SR, Fonseca R. 2005. Clinical implication of centrosome amplification in plasma cell neoplasm. Blood 107(9):3669-75.

Dodson H, Bourke E, Jeffers LJ, Vagnarelli P, Sonoda E, Takeda S, Earnshaw WC, Merdes A, Morrison C. 2004. Centrosome amplification induced by DNA damage occurs during a prolonged G2 phase and involves ATM. Embo J 23(19):3864-73.

Fernandez-Gonzalez R, Barcellos-Hoff MH, Ortiz de Solorzano C. 2004. Quantitative Image Analysis in Mammary Gland Biology. J Mammary Gland Biol Neoplasia 9(4):343-359.

Fukasawa K, Choi T, Kuriyama R, Rulong S, Vande Woude GF. 1996. Abnormal centrosome amplification in the absence of p53. Science 271:1744-7.

Fukasawa K, Wiener F, Vande Woude GF, Mai S. 1997. Genomic instability and apoptosis are frequent in p53 deficient young mice. Oncogene 15(11):1295-302.

Goepfert TM, McCarthy M, Kittrell FS, Stephens C, Ullrich RL, Brinkley BR, Medina D. 2000. Progesterone facilitates chromosome instability (aneuploidy) in p53 null normal mammary epithelial cells. FASEB J 14(14):2221-2229.

Hollstein M, Sidransky D, Vogelstein B, Harris CC. 1991. p53 mutations in human cancers. Science 253(5015):49-53.

Li JJ, Weroha SJ, Lingle WL, Papa D, Salisbury JL, Li SA. 2004. Estrogen mediates Aurora-A overexpression, centrosome amplification, chromosomal instability, and breast cancer in female ACI rats. PNAS 101(52):18123-18128.

Lingle WL, Barrett SL, Negron VC, D'Assoro AB, Boeneman K, Liu W, Whitehead CM, Reynolds C, Salisbury JL. 2002. Centrosome amplification drives chromosomal instability in breast tumor development. PNAS 99(4):1978-1983.

Maxwell CA, Keats JJ, Belch AR, Pilarski LM, Reiman T. 2005. Receptor for hyaluronan-mediated motility correlates with centrosome abnormalities in multiple myeloma and maintains mitotic integrity. Cancer Res 65(3):850-60.

Maxwell CA, Rasmussen E, Zhan F, Keats JJ, Adamia S, Strachan E, Crainie M, Walker R, Belch AR, Pilarski LM, Barlogie B, Shaughnessy J, Jr., Reiman T. 2004. RHAMM expression and isoform balance predict aggressive disease and poor survival in multiple myeloma. Blood 104(4):1151-8.

Mowat M, Cheng A, Kimura N, Bernstein A, Benchimol S. 1985. Rearrangements of the cellular p53 gene in erythroleukaemic cells transformed by Friend virus. Nature 314(6012):633-6.

Pihan GA, Purohit A, Wallace J, Knecht H, Woda B, Quesenberry P, Doxsey SJ. 1998. Centrosome defects and genetic instability in malignant tumors. Cancer Res 58(17):3974-85.

Pihan GA, Wallace J, Zhou Y, Doxsey SJ. 2003. Centrosome Abnormalities and Chromosome Instability Occur Together in Pre-invasive Carcinomas. Cancer Res 63(6):1398-1404. 
Raman S, Parvin B, Maxwell CA, Barcellos-Hoff MH. 2006. Geometric Approach to Segmentation and Protein Localization in Cell Cultured Assays. J Microsc (in press).

Ridler T, Calvard S. 1978. Picture threshholding using an iterative selection method. IEEE Trans on Systems, Man, and Cybernetics. SMC-8(8):630-2.

Salisbury JL, D'Assoro AB, Lingle WL. 2004. Centrosome amplification and the origin of chromosomal instability in breast cancer. J Mammary Gland Biol Neoplasia 9(3):275-83.

Zack GW, Rogers WE, Latt SA. 1977. Automatic measurement of sister chromatid exchange frequency. J Histochem Cytochem 25(7):741-53. 